\title{
LENGUA Y DIALECTO EN LA NOVELA ITALIANA CONTEMPORÁNEA
}

\author{
Manuel Carrera Díaz - Giorgia Marangon \\ Universidad de Sevilla
}

\begin{abstract}
This paper focuses on the use of dialect in the contemporary Italian novel. Many novels published in Italian in recent years make use, to a greater or lesser extent, of terms, expressions, or fragments of dialectal origin. The analysis tries to establish the place of those elements in the text and their role in the economy of the narrative. Finally, this paper considers the reasons for the use of such dialectal elements in the context of these works written in Italian.
\end{abstract}

\section{INTRODUCCIÓN.-}

La presencia del dialecto en el tejido narrativo en lengua italiana es uno de los elementos más llamativos de la forma expresiva de la novela italiana contemporánea. Es, en efecto, frecuente que, incluso en las novelas italianas publicadas en estos últimos años, aparezcan palabras, frases o incluso largos párrafos en dialecto, en uno de los numerosos dialectos italianos, pese a que la lengua de base en la que está escrito el resto del texto sea el italiano estándar.

Visto desde la perspectiva de los sistemas literarios que, como el escrito en español, se valen de una lengua "fuerte", que ha dejado tradicionalmente poco espacio a otros códigos lingüísticos, la cuestión no deja de parecer sorprendente. Pero desde la perspectiva italiana esta característica se explica más fácilmente: sus raíces hay que buscarlas en la excepcionalidad de la policéntrica historia de Italia, que, como advierte G. L. Beccaria, es un "paese di secolare tradizione comunale e regionale dove l'esito dialettale ha potuto costituire non già un paragrafo stravagante e marginale, ma una variante equipollente degli esiti della lingua" . Es cierto que, cuando se habla de "literatura italiana", ordinariamente se piensa en la escrita en lengua italiana, pero no es menos cierto que en pocas culturas como la italiana han estado tan cerca lengua y dialecto. En una reciente entrevista, el conocido lingüista Manlio Cortelazzo respondía con estas palabras a la pregunta de cuál es la diferencia entre lengua y dialecto: "Per un linguista, nessuna. La lingua assume uno status superiore solo per ragioni assolutamente extralinguistiche. Usiamo un'immagine: la lingua è un dialetto dotato di ambasciatore, passaporto ed esercito. Il veneto, per esempio, avendo questi tre requisiti poteva essere considerato una lingua. Poi li ha persi, e con essi anche lo status. Il dialetto è la lingua del cuore, quella che ti hanno insegnato la mamma e gli amici e che ti viene spontanea. La poesia dialettale oggi in Italia è considerata la migliore, proprio

\footnotetext{
${ }^{1}$ G. L. Beccaria (ed.) Letteratura e Dialetto (Bologna 1975) p. 1.
} 
perché non consumata da sette-otto secoli di scrittura. Nel dialetto rimane una chiarezza di espressioni, ed è un privilegio ma soffre dell'omologazione e questo è un guaio. Potrà resistere ma non per tanto"

Como recuerda P. De Meijer³, ya Bachtin había advertido hace tiempo que una de las características de la prosa novelesca es precisamente la pluralidad no sólo de voces y de discursos, sino también de lenguas. Pero si esto es cierto a nivel general, parece claro que "nella realtà italiana il plurilinguismo reale si manifesta in modo particolarmente sensibile, mentre dall'altra parte la lingua italiana costituisce un elemento unificante in una realtà che anche per molti altri aspetti è tutt' altro che unitaria".

Naturalmente, no se trata de un hecho nuevo o reciente 4 . La literatura dialectal tiene en Italia una amplia tradición. Ya desde finales del siglo XVI florece en este país, integrándose en la tradición áulica, una original producción literaria dialectal, sobre todo bajo las formas de la poesía amorosa, satírica y burlesca, que, según variantes diversas, seguirá desarrollándose en los siglos posteriores, para convertirse en el XIX, con Carlo Porta y G. G. Belli, en expresión profunda de la revolución romántica, evolucionando posteriormente bajo las poéticas del naturalismo y el realismo y alcanzando, sobre todo en el ámbito poético, un extraordinario vigor en el siglo $\mathrm{XX}^{\mathrm{s}}$.

Pero es curioso que, como sigue advirtiendo el propio De Meijer, la relación del dialecto con el género de la novela tiene unas características especiales. En los distintos dialectos de Italia se han compuesto poemas, textos teatrales o narraciones breves, pero no auténticas novelas. En este género literario, el dialecto tiene siempre una presencia excepcional, limitada cuantitativamente, constituyéndose siempre como forma lingüística extremadamente expresiva pero subordinada a la formulada en lengua italiana.

\section{DiAlecto y lengua en LA NOVELA ACTUAL。-}

Hemos pretendido conocer al menos de manera aproximada cómo es al respecto la situación actual, en las novelas publicadas en estos últimos años. No está de más recordar, como es bien sabido, que hoy en día el proceso de italianización, es decir, la difusión capilar de la lengua italiana en todo el espacio físico de este país, es prácticamente

${ }^{2}$ Il Gazzettino, 3 de diciembre de 2000, p. 5. Véase también al respecto G. Lepschy, "Dialect and language: a brief introduction", en Emanuela Tandello-Diego Zancani (a cura di), Italian Dialects and Literature. From the Renaissance to the Present (London 1996), pp. 1-6.

${ }^{3}$ P. De Meijer, "La prosa narrativa moderna”, en Letteratura Italiana (Torino 1984), vol. III, p. 771.

${ }^{4}$ Véanse al respecto, además de la obra ya citada de G. L. Beccaria, G. Luti, "Lingua e dialetto", en Narrativa italiana dell'Otto e Novecento (Firenze 1964); C. Segre, Lingua, stile e società. Studi sulla storia della prosa italiana (Milano 19763); C. Dionisotti, Geografia e storia della letteratura italiana (Torino 1977); A. Stussi, Lingua dialetto e letteratura (Torino 1993); I. Paccagnella, "Uso letterario dei dialettti", en L. Serianni-P. Trifone, Storia della lingua italiana (Torino 1993-91), vol. III.

${ }^{5}$ Respecto a la poesía dialectal italiana en el siglo XX, véanse, por ejemplo, además del mencionado volumen de Tandello-Zancani, F. Brevini, Le parole perdute. Dialetti e poesia nel nostro secolo (Torino 1990) y Poeti dialettali del Novecento (Torino 1987), así como H. W. Haller, The Hidden Italy. A Bilingual Edition of Italian Dialect Poetry (Detroit 1986) y A. Dolfi, Poesia dialettale e poesia in lingua nel Novecento. Intorno all'opera di Marco Pola (Milano 1994) 
completo: ha aumentado en progresión geométrica el número de hablantes originariamente dialectófonos que son capaces de expresarse en la lengua nacional, y el italiano hoy, para la gran mayoría de los italianos, no es ya sólo una lengua escrita o propia de ámbitos específicos, sino también usualmente hablada por toda la comunidad circundante. Cabría esperar, pues, que la utilización incluso ocasional del dialecto como forma expresiva literaria hubiese sufrido una fuerte reducción, cercana a su práctica eliminación ${ }^{6}$. ¿Cuál es, en realidad, y dada esta fácilmente constatable situación lingüística, la relación lenguadialecto en la producción novelística de los últimos años?

Nuestra investigación al respecto, como se podrá observar, es más bien artesanal y un tanto aleatoria, pero confiamos en que también sea, al menos en cierta medida, representativa. Hemos tomado en consideración 33 novelas publicadas en Italia entre 1992 y 1999, equilibradamente distribuidas en el tiempo a lo largo de este período 7 . No se trata de una elección puramente casual: el grupo dominante está constituido por novelas finalistas del premio "Un Autore per l'Europa", otorgado anualmente por el Ayuntamiento de Alassio. Y ello porque en nuestra selección hemos pretendido huir de productos literarios excesivamente localistas, prefiriendo basarnos en obras de alcance nacional, susceptibles de recibir una aceptación y consenso generalizados.

El resultado, que podemos anticipar en pocas palabras, ha sido éste: de las 33 novelas objeto de análisis, 20 no tienen presencia expresa de elementos dialectales, mientras que 13, por el contrario, se valen, en mayor o menor medida, del dialecto. No se trata, pues, de un porcentaje despreciable: más de un tercio de las novelas publicadas en ese período, si nuestra cuantificación pudiera extrapolarse, se valdrían ocasionalmente del dialecto como forma expresiva. En las páginas que siguen trataremos de explicitar los modos y formas de esa presencia dialectal.

\section{FORMAS DE LA PRESENCIA DEL DIALECTO.-}

\subsection{Tipología de la "novela con dialecto".}

No hay una tipología precisa de la novela con contenidos expresivos dialectales. Ni por su argumento, ni por el origen geográfico de su autor, ni por su extensión o cualquier otra característica objetivable puede decirse que tengan algo en común, como no sea, precisamente, el uso minoritario de un dialecto. Estas trece novelas de las que hablaremos a continuación, y cuya relación completa damos al final de este trabajo, son completamente distintas entre sí, sin que puedan relacionarse, insistimos, por rasgos formales o de contenido.

\footnotetext{
${ }^{6}$ Sobre la aparente paradoja constituida por la contradicción existente entre el declive del dialecto en el uso común y su reafirmación en el ámbito literario, advierten E. Tandello y D. Zancani: "Among the paradoxes of contemporary Italy the decline of dialects in every day usage is accompanied by a resurgence in their use in literature" (op. cit. p. vii.)

${ }^{7}$ Sobre la narrativa anterior a esa nuestra fecha inicial véase, en este mismo sentido, H. Haller, "La presenza dialettale nella nuova narrativa", en F. Musarra, S. Vanvolsem y B. Van Den Bossche (eds.), Rinnovamento del codice narrativo in Italia dal 1945 al 1992. Atti del Convengo internazionale (Leuven, Louvain-la-Neuve, Namur, Bruxelles, 3-8 maggio 1993), (Roma 1995), vol. II, 243-256. De este mismo autor véase también "Literature in dialect and dialect in literature: a sociolinguistic perspective”, en E. Tandello-D. Zancani, cit., pp. 73-80.
} 


\subsection{El nivel cuantitativo}

El nivel cuantitativo de la presencia del dialecto varía, obviamente, en manera vistosa de una novela a otra. Se va de un mínimo que parece puramente testimonial y episódico ( $F$. Tomizza, Franziska) a textos en los que el dialecto se mezcla constantemente con el italiano, casi como si se tratase de la misma lengua (L. Pariani, La perfezione degli elastici [e del cinema]), pasando por novelas en las que aparece en forma salteada (S. Maldini, La stazione di Varmo), en las que va marcando de manera casi rítmica casi todo el relato, en alternancia con el italiano (G. Montesano, Nel corpo di Napoli), o en las que aparece evidenciado y aislado en largos párrafos (G. Rugarli, L'infinito forse).

\subsection{Las zonas de presencia del dialecto}

Es interesante preguntarse cuáles son las zonas de presencia del dialecto, analizando las variantes de la forma narrativa. A este respecto, notaremos que:

\subsubsection{Aparece en el texto narrado en obras como las de}

\section{- S. Vassalli:}

"Suo padre era lo scarpér cioè il calzolaio di Casal” (p. 19)

"Si videro venire incontro tre ferali portati da altrettanti uomini mascherati con larve di panno bianco" (p. 39)

"Responsabili di tutto erano i marici, cioè i sindaci dei villaggi" (p. 129)

- F. Tomizza:

"Il sindaco detto zumpano" (p. 27)

"Il corpiccino insaccato nella rigida plàtizza" (p. 30)

“La piastra dell'enorme spàrherd" ( p. 50)

- G. Rugarli:

Introduce largos párrafos en romanesco (pp. 30-32), napolitano (pp. 69-71), siciliano (pp.236-238), milanés (pp. 270-272), véneto (pp. 308-310) e incluso inglés (pp. 103-105).

- S. Givone:

"Due arbàrete di capre in gelatina" (p.11)

'Non così i figli. Da una parte cui d'la casina, quelli della cascina, e dall' altra cui d'la ca', quelli della casa" (p. 25)

"Vado al ruscello. A prendere le sgrape" (p.70)

- L. Pariani:

"Quando si è avvicinato al letto della Gostìna, c'era una tale spüzza di orina e di diarrea che gli è venuto ingóssa" (p. 19)

3.3.2 Aparece en el texto dialogado en la mayoría de las novelas: 
- R. Barbolini:

"Li mè fioli son tanti malahim!" (p. 123)

- S. Maldini:

“Ma e jé pur simpri une biele bighe (ma è pur sempre un bel cazzo) (p. 35)

"Ninine è laconica. "El gjal c'al lavore al a fan (il gallo che lavora ha fame) dice". E poi aggiunge: Normale. "Indulà c'al è gjal e son lis gjalinis (dove c'è il gallo ci sono le galline)" (p. 135)

- G. Bettin:

“Là inte! Là inte!” (p. 14)

- S. Givone:

"Prima di bere, aveva emesso la sentenza: "L ê nen cuma ca dêै". Non è come debe". (p. 21)

"I mör", muoio, si è limitato a dire (p. 71)

- L. Pariani:

"L'é méj sarâ sü, pütost ca in strâ" dice sempre sua madre (p. 20)

- G. Montesano:

"Già mó sono un cadavere. Nu muorto! (p. 13)

- G. Pansa:

"Bisogna vardarse da i denti de '1 can e da ci g'ha sempre il rosario in man" (p. 96)

"La politica l'è ancó pan e fame doman (oggi sembra darti il pane ma domani ti offrirà soltanto fame e fatica) (p. 105)

3.3.3 Un territorio muy favorable para la aparición del dialecto es el de los refranes y proverbios:

- S. Givone:

“Cul ca pöl nen dí la verità, ca staga citu, ma cul ca pöl nen stè citu, ca diga la verità" (p. 35)

- S. Maldini:

“Indulà c'al è el gial e son lis gjalinis" (p. 135)

- G. Pansa:

"Ndo gh'e braga, dòna no paga" (p. 10)

"Bela in facia, bruta in culo" (p. 19) 
"I brodi longhi no i fa ben gnànca a i malà"(p. 37)

\subsection{Longitud de las secuencias lingüísticas dialectales}

La longitud de las secuencias lingüísticas dialectales es muy variable. Tenemos, en síntesis, los siguientes casos:

3.4.1 La palabra aislada, que aparece junto con otras en una especie de diccionario o glosario que remite a otros mundos:

- G. Bettin:

"Per vedere l'enrosadira" (p. 19)

- S. Givone:

"Lo trattano come un giròni qualsiasi... “ (p. 59)

- G. Pansa:

"A volte immagino: gli canterò il tantum ergo, lo ciaparò per i corni" (p. 51)

3.4.2 Sintagmas dialectales enlazados con secuencias italianas:

- L. Pariani:

"Ma perché lo tratti così bene 'sto bausciâtu? (p. 21)

"Sacramèmball, son tre anni che mio fratello a l'è part̂, capite? E ora lei sta per marâss (p. 27)

- G. Pansa:

“Sì, sono calda come la mare de'l fogo. Però non è colpa del sole" (p. 33)

- S. Givone:

"Noi siamo men che niente, 'na creva... (p. 43)

3.4.3 A veces aparecen secuencias de frases o párrafos en dialecto:

- L. Pariani:

"Par ul mé instriaméntu -al dìs l'umbriûn-a gh'avéu da bisògn da trua vüna ca ma vuréa bén, anca sa mi a séru minga bel" (p. 13)

- M. Maggiani:

“Ciau Giaguaro, comme l'è? E ammia in po'chi Tirreno, che giubunetto che u l'ha u Minu" (p. 207)

\section{LAS FORMAS DE LA INTEGRACIÓN.}

Es interesante también comprobar en qué forma y medida el elemento dialectal se integra, implícita o explícitamente, en el tejido en lengua italiana: 
4.1 A veces se hace referencia explícita al hecho dialectal:

- G. Pansa:

"[Vorrei] mangiarti. Vuoi che te lo dica nel mio dialetto? Mi te sbranarìa" (p. 16)

- Guccini-Macchiavelli:

"Era dentro il fiume e si teneva a galla aggrappato a uno stropello..." "Parla italiano. Cos'è uno stropello?" "Come lo chiamate, voi? Un ramo di quelli che nascono sulla riva del fiume e che servono per fare i cesti..." "Va bene, un giunco, allora?

4.2 En otras ocasiones, el vocablo se mimetiza como si fuera plenamente italiano:

- G. Bettin:

"Il mare si udiva muggire attorno ai fariglioni" (p. 26)

- G. Pansa:

"E anch'io mi sento tutta saccagnata" (p. 158)

- D. del Giudice:

"E poi vedresti soltanto 'sto strunzo" (p. 85)

"Tanto t' acchiappo, tanto t'accoppo" (p. 86)

4.3 Muy a menudo, la secuencia dialectal aparece claramente evidenciada mediante el uso de la cursiva:

- S. Maldini:

"Tu pûs crodi benedette" (tu puoi credere benedetta (p. 9)

- S. Givone:

"La mirauda", regina delle bisce (p. 5)

"Gent ca crêd 'n nient" (gente che non crede a niente) (pp. 23-24)

- S. Vassalli:

"Le finestre del suo tabiă" (p. 23)

4.4. O se presenta delimitada entre comillas, pero como si fuera texto italiano:

- R. Barbolini:

"Li gnascririm se cheven tuti i so vogli" (p. 125)

- G. Montesano:

"Senza spargimento di sangue non c'è remissione dei peccati. "Accussì è 'a legge e 'a legge è vendetta" (p. 47) 
4.5 O, finalmente, no aparece evidenciada en absoluto, y se presenta al mismo nivel textual que el tejido italiano. Los ejemplos más significativos a este respecto nos vienen de L. Pariani:

"L'umbriûn, l'orco, il magnàno-padelle delle favole che mi contavano da piccola, ul scimbióttu mostruoso con la scüffia viola". (p. 13)

"Sul sentiero la sua ombra è molto più piccola di quella da chél grand'e ciùla dul Biâs". (p. 19)

\section{TRAdUCCIÓN O INTEGRACIÓN}

Por lo que se refiere al nivel de comprensión por parte del lector, la actitud del autor puede ser doble: suministrar la traducción del texto dialectal, o dejarlo tal cual, sin mayores aclaraciones. En el primer caso, el de la traducción suministrada, los casos pueden ser estos:

5.1 Ofreciendo una explicación del equivalente:

a) En forma directa:

- Vassalli:

'l' adunata delle cernide, cioè della milizia territoriale per la difesa della valle" (p. 91)

"Reggendo il feral cioè la lanterna" (p. 39)

b) En forma indirecta:

- G. Pansa:

“Ndo gh'è braga, dòna no pagal, se c'è un maschio con le brache è lui che paga, non la ragazza" (p. 10)

5.2 Suministrando la traducción inmediata y literal, generalmente entre paréntesis:

- S. Vassalli:

“Gusele (spille)... piroi (oreochini)” (p. 31)

- S. Maldini:

"In dutis lis çiasis a jé plui di una puarta" (in tutte le case c'è più di una porta) (p. 35)

5.3 Otros autores, sencillamente, no ofrecen traducciones ni suministran la menor ayuda al lector. Casos paradigmáticos, en el corpus que hemos examinado, son los de L. Pariani y G. Rugarli.

\section{LAS RAZONES DEL DIALECTO}

Una cuestión digna de estudio sería la de la determinación del grado de adecuación lingüística de estas secuencias dialectales: el problema podría platearse y resumirse en las siguientes preguntas: ¿el dialecto empleado en estas novelas refleja efectivamente las construcciones reales de las hablas dialectales? ¿Se trata de dialectos reales o literarios? ¿Hasta qué punto son reales y hasta qué otro inventados o reelaborados literariamente? 
Naturalmente, en algunos casos caben pocas dudas. Cuando, por ejemplo, Vassalli constela su texto con vocablos dialectales aislados, no hay motivos para dudar que se trate de correspondencias lingüísticas reales. Pero cuando el dialecto alcanza como mínimo el nivel de frase, es decir, cuando hay una elaboración lingüística que va más allá del simple glosario, la duda puede insinuarse en la mente del lector. Algunos lectores ligures, por ejemplo, nos informan de que el dialecto que usa Maurizio Maggiani en La regina disadorna no suena exactamente como ligur en todos sus extremos. Daniele del Giudice, en uno de los relatos breves del volumen Mania, se sirve de un napolitano che en realidad no va más allá de algunas expresiones conocidas por todos los italianos (isto strunzo, sei piccirillo) y de fragmentos textuales de ciertas muy populares canciones napolitanas. Giampaolo Rugarli, en el epílogo de su novela, manifiesta su agradecimiento a los traductores que han preparado las versiones de sus textos en los distintos dialectos ${ }^{8}$. Es decir, que en estas novelas no sólo se utiliza el dialecto, sino que se hace uso de él incluso cuando no se lo conoce, o cuando se lo conoce a un nivel más bajo del que tiene un hablante nativo. Razones más que suficientes, pues, para preguntarse, como hacíamos al comienzo de este apartado, por el grado o nivel de adecuación lingüística de estas secuencias. Cuestión que, obviamente, no podemos tratar en el ámbito de este breve análisis.

Más pertinente aquí es que nos preguntemos por qué se usa el dialecto en el seno de un campo textual que se vale como lengua básica de la nacional. Aunque, tratándose de la literatura escrita en Italia, quizás fuese más legítima la pregunta contraria: ¿por qué no debería utilizarse el dialecto? Como señalaba M. Cortelazzo en el fragmento de la entrevista que recogemos al comienzo de este trabajo, para muchos italianos, sobre todo de cierta edad, "el dialecto es la lengua del corazón, la que te han enseñado tu madre y tus amigos y que te sale espontáneamente". Hay un componente, pues, de cercanía mental y sentimental con el dialecto que puede en parte explicar la sorprendente facilidad y normalidad con la que los escritores italianos lo insertan en el seno del texto italiano?.

En algunos casos la función del dialecto parece puramente documental, como en las novelas de S. Vassalli o F. Tomizza: en dialecto tiende a recogerse sólo lo peculiar del ambiente descrito, y de ahí esa distribución dialectal en forma prevalente de glosario. En otros, parece deberse al deseo de fidelidad en la caracterización de un ambiente o un personaje, del que se transcribe su forma de expresión (G. Pansa, S. Maldini). Algunas

\footnotetext{
${ }^{8} \mathrm{Y}$ explica cuál ha sido su intención al respecto: "I filologi forse eccepiranno sulla purezza e sulla correttezza dei dialetti: in realtà non ho inteso resuscitare linguaggi purtroppo condannati a morte, bensì cogliere alcuni profumi dell'universo italiano, tanto dissimile, tanto variegato. Tutti i profumi hanno uguale nobiltà. La loro ricchezza non inficia la validità del progetto rinascimentale, semmai vale ad esaltarla, perchè suggerisce tolleranza, comprensione, rispetto" (op. cit., pp. 329-330).

${ }^{9}$ Entre los autores que estudiamos, así lo reconoce explícitamente, por ejemplo, G. Bettin: "La tua lingua è stata la mia solo per poco, quand'ero bambino. Lo è stata sempre meno, poi. Ne cercavo un'altra, che non fosse inerme, umiliata come la tua -la nostra- mi sembrava. Mi suonava debole, sottomessa e volevo impararne una nuova, che non fosse una lingua sconfitta. Ora so che era dolce, e che era mite, quella lingua, e so che se anche vi eccheggiava un'ansia, un timore, la sosteneva lo sforzo di vivere, di resistere. In quella fatica, che infine ti ha piegata, contrastavi un destino brutale, già scritto. Per questo, nel tempo, la tua lingua ha continuato a parlare" (op. cit., p. 125).
} 
modalidades de uso dejan entrever una intención satírica o irónica, jugándo con el contraste entre un plano de referencia en lengua italiana y un plano de desfase en dialecto ( $G$. Montesano). En otros autores, el dialecto se presenta como un elemento de rotura del molde lingüístico "comprensible", como es el caso del texto de L. Pariani. Se va, pues, desde lo puramente documental a lo decididamente expresionista, en una multiplicidad de funciones que acreditan al dialecto como una fuente de recursos expresivos enormemente variada y plural, posibilitando, como sostiene acertadamente Haller, esa "cointinuing plurality of Italian languages and literatures in a society that continues to be multilingual"10.

\section{LISTA DE OBRAS ESTUDIADAS}

R. BARBOLINI, Il punteggio di Vienna (Milano 1995)

G. BETTIN, Nemmeno il destino (Milano 1997)

D. DEL GIUDICE, Mania (Torino 1997)

S. GIVONE, Favola delle cose ultime (Torino 1998)

F. GUCCINI-L. MACHIA VELLI, Macaroni (Milano 1997)

M. MAGGIANI, La regina disadorna (Milano 1998)

S. MALDINI, La stazione di Varmo (Venecia 1994)

G. MONTESANO, Nel corpo di Napoli (Milano 1999)

G. PANSA, Siamo stati così felici (Milano 1995)

L. PARIANI, La perfezione degli elastici (e del cinema) (Milano 1997)

G. RUGARLI, L'infinito, forse (Alessandria 1995)

F. TOMIZZA, Franziska (Milano 1997).

S. VASSALLI, Marco e Matthio (Torino 1992)

${ }^{10}$ Op. cit. p. 80. 